\title{
A Rule-Based Expert System to Decide on Direction and Speed of a Powered Wheelchair
}

\author{
David A Sanders TD VR \\ School of Engineering \\ Portsmouth University, UK \\ david.sanders@port.ac.uk
}

\author{
Alexander Gegov \\ School of Computing \\ Portsmouth University, UK \\ alexander.gegov@port.ac.uk
}

\author{
Malik Haddad \\ School of Engineering \\ Portsmouth University, UK \\ malik@malikhaddad.com
}

\author{
Favour Ikwan \\ School of Engineering \\ Portsmouth University, UK \\ Favour.Ikwan@myport.ac.uk
}

\author{
David Wiltshire \\ School of Engineering \\ Portsmouth University, UK \\ david.wiltshire@port.ac.uk \\ Yong Chai Tan \\ Faculty of Engineering \& Built \\ Environment \\ SEGi University, Malaysia \\ tanyongchai@segi.edu.my
}

\begin{abstract}
Some rule based techniques are presented that can assist powered wheelchair drivers. The expert system decides on the direction and speed of their wheelchair. The system tends to avoid obstacles while having a tendency to turn and head in towards a desired destination. This is achieved by producing a new target angle as an extra input. Other inputs are from sensors and a joystick. Directions are recommended and mixed with user inputs from the joystick representing desired direction and desired speed. The rule-based system decides on an angle to turn the powered wheelchair and suggest it. Inputs from the joystick and sensors are mixed with the suggested angle from the Rule Based Expert System. A modified direction for the wheelchair is produced. The whole system helps disabled wheelchair users to drive their powered wheelchairs.
\end{abstract}

Keywords-Rule-based; decision; wheelchair; assist; powered; collision avoidance; driving

\section{INTRODUCTION}

This paper proposes a rule based expert system to assist with the control of a powered wheelchair. Powered wheelchairs are used by disabled people who cannot use a manual wheelchair because they can't rotate the wheels. A powered wheelchair can provide independence and freedom [1]. The methods and systems presented here will help more disabled people to drive powered wheelchairs. Knowledge about the environment around the wheelchair is provided by ultrasonic sensors so that the system can assist a disabled driver with avoiding obstructions and obstacles in their path. Knowledge of the direction towards a desired destination allows the system to tend to guide the wheelchair towards the destination.

Around seven million Americans use assistive mobility devices. There are about two million wheelchair and scooter users and another five million use other devices, for example walkers, canes and crutches [2]. Nearly one third of people using mobility devices require assistance from other people [1].

The predominant primary conditions for wheelchair and scooter users are stroke and osteoarthritis. Osteoarthritis is the main condition linked to using mobility devices [1], [2].
A powered wheelchair is usually used by people lacking mobility or dexterity because of shoulder, arm hand, or more widespread disability, and who do not have enough strength in their legs to use their feet to push a manual wheelchair. Powered wheelchairs can also provide tilt, recline and elevation, and bespoke functions for health and normal day-today functioning.

Powered wheelchairs can be categorised into four types: wheelchairs driven by their front, centre or rear wheels and four-wheel drive wheelchairs. They can also be categorised by their seating: (a) similar to a seat in a car and (b) with a slingstyle seat and frame.

A user of a wheelchair often controls their speed and direction with a joystick. If a user lacks the coordination to effectively use a joystick or if they can't use their hands or fingers then other input devices can be used (foot control, sip tubes/puff switches, or head or chin controllers, etc.).

Technology is allowing more and more people to use powered wheelchairs for both indoor and outdoor use.

They can include outdoor wheels and tires and powered wheelchairs can move at up to $6 \mathrm{mph}$. They can have extra wheels to provide stability, for example when outside and away from pavements and roads. Rear or mid wheel drive chairs are well-liked for use indoors and outdoors.

Users of powered wheelchair can spend a great deal of time in their wheelchairs, so they need to suit the environment where they are being used. Every wheelchair driver is unique and they need bespoke seats, arm rests and leg rests to provide comfort and stability. Powered seats, reclining and tilting backs, and electric leg rests are possible additions.

If a user has head injuries, neurological or physiological problems, or lacks special awareness then they might not be able to safely steer. Potential wheelchair users could be unable to avoid collisions or be blind, etc. Systems described here are helping these disabled wheelchair drivers to drive more safely. 
Controllers for wheelchairs are normally open-loop and drivers indicate desired speed and direction by positioning an input device (for example a lever or joystick) and the chair tends to travel at the desired speed along the desired route. Wheelchair users make corrections to evade obstacles. This paper describes how information from an input device can be processed and mixed with inputs from a sensor system and a target destination to assist a wheelchair user in steering their chair. Global planning and local planning combine inside a rule-based expert. The result provides drivers with assistance. A global path is mixed with local information from the sensor system [3].

Powered wheelchair navigation has been considered [4], [5]. Algorithms have typically been local and no attempt has been made to improve a system more globally. Obstacle avoidance has been considered [6] with local inputs from sensors [7].

Some research work has calculated initial wheelchair paths and then has locally modified them if an obstruction was perceived [3] but they have rarely been used successfully to assist wheelchair users. This paper describes how three inputs can be used with a local planner to drive the wheel motors. The three inputs are: on-board sensors, a joystick, and a global target destination. The powered wheelchair can react quickly to joystick movements but can also respond to any obstacles that might be detected ahead. The powered wheelchair tends to turn towards the global destination but can avoid obstacles along the way.

Huq et al. used a fuzzy context-dependent system to eliminate some limitations [8] by using a goal oriented navigation while avoiding obstacles. Genetic algorithms were mixed with Fuzzy logic to overcome some mapping difficulties and establish a local position [9]. Bennewitz \& Burgard presented random planning methods that could produce realtime paths in unknown environments [3], [10] that precisely followed trajectories [11]. Hwang \& Chang presented obstacle avoidance methods that used fuzzy decentralized sliding-mode control [12]. Song \& Chen solved some of the local minima problems and then improved on the well-known potential-field technique [7] and Nguyen et al produced Bayesian Neural Networks to avoid obstructions [13].

Techniques are presented here to partially optimise some minimum-cost paths. A joystick regulates direction and speed and AI systems provide input to modify them if necessary [14][17]. The system uses perception based rules that are similar to [35] and [3].

Calculations trade off the distance to objects against the length of a path. A steering angle is determined by rules and that is combined with input from the joystick. A new revised steering angle is created and that is used to drive the motors. The procedures were tested in simulation and with sensors mounted on a Bobcat II powered wheelchair (Fig. 1).

Many different sensors can be used to assist a powered wheelchair user to safely avoid obstacles [18]: infrared [21]; ultrasonic [20] or laser or structured light [19]. Global systems are tricky to use inside a building [22] but local sensors have been used such as: gyroscopes, odometers, tilt sensors and ultrasonics [23], [24]. Cameras are reducing in price but processing tends to be more complex [25]. Computers are getting more powerful and are also reducing in price [26]. That means that cameras are being used more often for applications. The best source of knowledge about what is required is still usually the disabled human driver, but reduced visibility or their disability can reduce their proficiency [27].

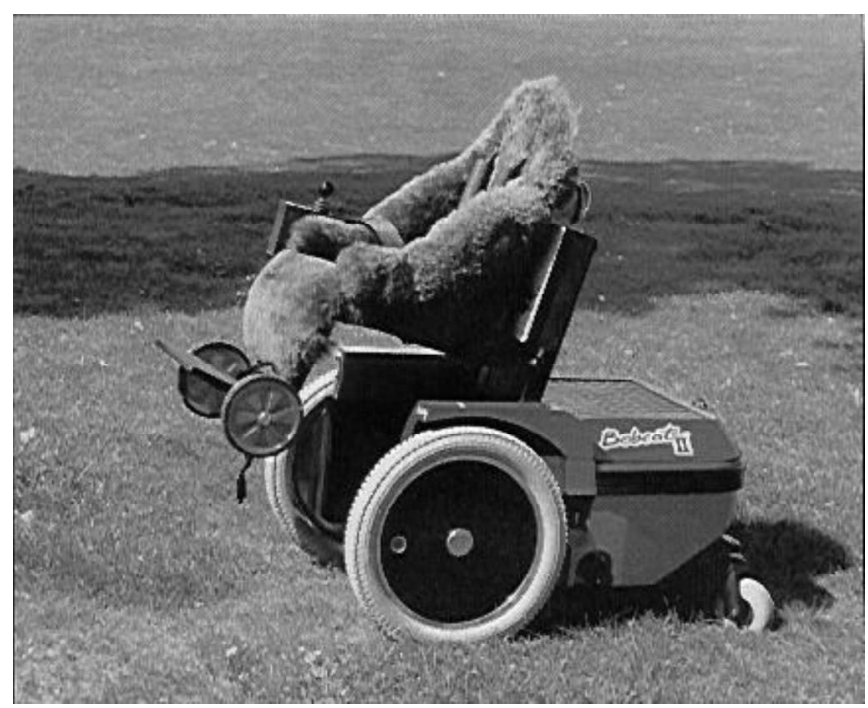

Fig. 1. A Bobcat II powered wheelchair.

Ultrasonics were selected because they are robust, cheap and simple [28]. The sensors and input from the joystick are described in Section II and then Section III describes wheelchair kinematics. Section IV describes the rules and the control methods and Section V presents some of testing and results. Section VI is a short conclusion.

\section{JOYSTICK AND SENSOR INPUTS}

\section{A. Ultrasonics}

The ultrasonic systems are like the systems described in [29]-[33], [35]. Sensors were mounted above each driving wheel. Distance to objects was measured using the time taken for pulses to reflect back from the obstacle to the receivers.

The wheelchair has a solid steel frame for strength and stability and is covered by a shell made of fibreglass. Trailing casters are at the back and large driving wheels are at the front. Each driving wheel had an ultrasonic sensor secured on the frame above it.

A joystick was usually connected straight to a wheelchair controller to steer the powered wheelchair. In the research presented in this paper, that direct connection between powered wheelchair and joystick was parted and instead, a computer was introduced in between the chair and joystick. The joystick input was then managed by the computer. The system could function in a choice of:

- Joystick input sent directly to controller.

- Joystick input modified by the computer to adjust direction and speed.

Three basic rules applied to modifying direction and speed: 
1) Overall control remained with the disabled wheelchair user.

2) Direction and speed were only adjusted when needed.

3) If a change in speed and direction was needed then the change applied was smooth.

Imaginary potential fields were placed around obstacles detected by the sensors [7], [23], [35]. If nothing was being sensed then a range-finder gradually increased the range of the sensors (by lengthening the ultrasonic pulses) until potential obstacles were detected so that the system provided warnings of likely difficulties ahead.

\section{B. Mapping the Environment}

Ultrasonics are often noisy and can provide incorrect readings. Because of that, Histogramic In-Motion Mapping was used to filter out any incorrect readings [35]. A volume ahead of the wheelchair was divided into a right-hand side and a left-hand side. A matrix was then established for each side and an overlapping volume with three elements in each matrix: IMMEDIATE, HALFWAY and OUTLYING.

If an obstacle was detected somewhere in front of the powered wheelchair then it was labelled as IMMEDIATE, HALFWAY or OUTLYING. Sensor beams over-lapped and bounded the volume ahead. The centre matrix denoted circumstances when both left and right sensors had detected something.

The volume ahead of the powered wheelchair was therefore represented by a 2-D 3x3 grid with nine elements:

\section{LEFT-HAND SIDE, MIDDLE, RIGHT-HAND SIDE}

$\mathrm{X}$

\section{IMMEDIATE, HALFWAY, OUTLYING}

If obstacles were sensed then associated element(s) in the grid were increased by a relatively large amount, for example 5 , to a max value of 15 . Other empty elements were reduced by a smaller amount (for example 2) down to zero. In that way, a straightforward histogrammic representation was created that represented a volume ahead of the powered wheelchair. When obstacles were sensed then the values of the associated cells swiftly increased. Random errors in any cells might fleetingly increase because of solitary misreads but they would then quickly reduce again. If obstacles were detected in an element but then they moved to another element, then the new element quickly increased in value. If an obstacle vanished from an element then its value decreased to zero. A reliable estimation of the range to an obstacle was arrived at within $<0.4$ seconds.

\section{Joystick}

A Penny \& Giles joystick was used to drive the powered wheelchair. The joystick had $2 \mathrm{x}$ potentiometers within it and joystick position was resolved with $2 \times \mathrm{A} / \mathrm{D}$ converters that were connected to the potentiometers. The data extracted from the joystick was effectively in a Cartesian coordinate system. The Cartesian coordinates were transformed to a polar coordinate system: $|\mathrm{J}| \angle \theta$. The speed demanded by the wheelchair driver was represented by the angle that the joystick moved away from a central location $|\mathrm{J}|$. The direction demanded by the wheelchair user was represented by $\angle \theta$.

The time that a joystick remained in a position gave a degree of confidence.

$$
|\mathrm{J}|=\sqrt{ }((\text { Alpha*Alpha })+(\text { Beta*Beta }))
$$

Where, Alpha and Beta are the Cartesian co-ordinates.

Histogrammic mapping worked like a pseudo-integrator. When joysticks were kept in a steady position and orientation then the associated element increased over time and other elements reduced. An element with the greatest value represented the stick orientation and position.

$|\mathrm{J}|$ and $\theta$ represented desired direction and speed. Confidence in that desired direction and speed was also recorded. Each cell in the array had two values:

- "AngularConf" represented how steadily the joystick was held in a position.

- "SpeedMag" represented desired speed.

JoyIn was the joystick input to the rule based expert system. JoyIn represented the confidence-level for the disabled driver intentions. JoyArray calculated which element represented the joystick and AngularConf increased. Other empty element reduced in value. Histogram elements reduced quickly but increased more slowly. JoyArray elements increased to a max value in $<0.4 \mathrm{~s}$ and reduced to zero in $<130$ ms.

The rates to increase or decrease in value were verified experimentally. Individual rates could be established for individual users or for particular tasks.

\section{KINEMATICS OF A BOBCAT II POWERED WHEELCHAIR}

Wheelchair kinematics are shown in Fig. 1. A Bobcat II powered chair has two bigger driving wheels at the front of the chair that are driven independently. Driving the front wheels at different speeds allows a disabled wheelchair user to control the direction and speed of a chair. If wheel radius is $r$ then wheel diameter is $2 \mathrm{r}$. Using the notation and symbols of [3] and [35], the $2 \times$ front wheels were distance $\mathrm{W}$ apart. The centre of gravity is at $\mathrm{C}$ and $\mathrm{P}$ defines the intersection of a line through the wheel axis and another line through the centre of the chair. D is the gap between $\mathrm{P}$ and $\mathrm{C}$. then,

If there is not any slip between the wheels and the floor

$$
\begin{aligned}
& v_{\text {tang }}=1 / 2\left(v_{\text {right }}+v_{\text {left }}\right) \\
& \omega_{\text {tang }}=1 / \mathrm{W}\left(v_{\text {right }}-v_{\text {left }}\right) \\
& v_{\text {right }}=r \omega_{\text {right }} \text { and } v_{\text {left }}=r \omega_{\text {left }}
\end{aligned}
$$

where, $\mathrm{v}$ is the linear velocity and $\omega$ is angular velocity.

Global position of the chair in global coordinates is [O X Y] using vector notation. So that:

$$
\boldsymbol{q}=\left[\mathrm{x}_{\mathrm{C}} \mathrm{y}_{\mathrm{P}} \theta\right]^{\top}
$$




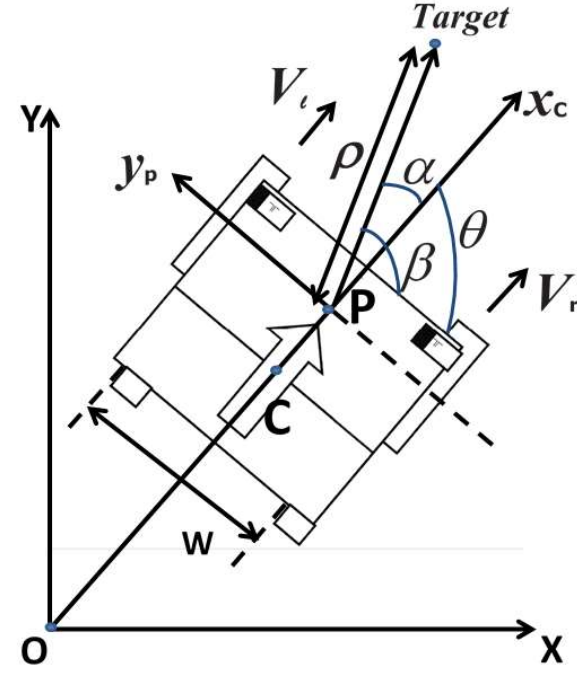

Fig. 2. Wheelchair kinematics.

Where, $x_{C}$ and $y_{P}$ are the global coordinates of $P . \quad \theta$ is the orientation of the local coordinate frame, that is $\left[P x_{C} y_{P}\right]$ in Fig. 2 with respect to the horizontal axis. The coordinates of the chair are defined by (5). The body of the chair is assumed as rigid and the driving wheels are assumed not to slip. So the chair can only move normal to the axis of the driving wheels. The velocity at the point of contact between a wheel and the (orthogonal to the wheel plane) is zero.

$$
\left(d y_{p} / d t\right) \cos \theta-\left(d x_{C} / d t\right) \sin \theta-d \theta / d t=0
$$

Kinematics restrictions are not time dependent and are

$$
\begin{aligned}
& \mathrm{A}^{\top}(\boldsymbol{q}) \mathrm{d} \boldsymbol{q} / \mathrm{dt}=0 \\
& \text { Where, } \mathrm{C}^{\top} \mathrm{A}(\boldsymbol{q})=0
\end{aligned}
$$

and $A(\boldsymbol{q})$ is an input matrix associated with the constraints.

$C(\boldsymbol{q})$ is a full-rank matrix formed by a set of linearly independent vector fields covering the null space of $A^{\top}(\boldsymbol{q})$ and $v_{\text {tang }}$ is a vector time function obtained from (7) and (8) for time $t$.

$$
\mathrm{D} \boldsymbol{q} / \mathrm{dt}=\mathrm{C}(\boldsymbol{q}) \boldsymbol{v}_{\text {tang }}
$$

The constraint matrix in (6) for the powered wheelchair is

$$
\mathbf{A}^{\top}(\boldsymbol{q})=[-\sin \theta \cos \theta-\mathrm{d}]
$$

and

$$
\boldsymbol{v}_{\text {tang }}=\left[\begin{array}{ll}
v & \omega
\end{array}\right]^{\top}
$$

Where, $\mathrm{v}$ is the linear velocity and $\omega$ is the angular velocity of meeting point $\mathrm{P}$ (down the chair axis).

So, the kinematics described by (9) can be placed into a $\mathrm{d} q / \mathrm{dt}$ matrix.

If the chair only moves forward then $v=-v$, ang and a streamlined matrix can represent the wheelchair.
A controller generated the steering angle and wheel velocities where Angle $=\left(v_{\text {left }}-v_{\text {right }}\right) / \mathrm{W}$, to steer the chair along a desired path.

\section{CONTROL AND THE RULES}

The linear and angular velocities ( $v$ and $\omega$ ) to move the chair were calculated to take the chair from the current position $\left(\rho_{0} \alpha_{0} \beta_{0}\right)$ to a new desired target position. Considering linear control [34],

$$
v=K_{\rho} \rho
$$

and,

$$
\omega=K_{\alpha} \alpha+K_{\beta} \beta
$$

A matrix could depict the closed-loop system to drive the wheelchair to the destination $(\rho, \alpha, \beta)=(0,0,0)$.

Code for an overdamped controller was written and simulated successfully before being downloaded to the powered wheelchair systems. Joystick input was combined with the input from ultrasonic sensors using expert rules that were calculated in order to assist the user in avoiding obstacles. The initial set of rules were enhanced and upgraded later by including a global target destination (a final target destination or via point) so disabled wheelchair users could tend to follow an efficient global path. The revised rules combined five inputs in an effort to avoid obstacles and follow a more global path (Fig. 4). They were (Fig. 3):

- Demanded steering angle (from a joystick).

- Distance to objects detected to the right.

- An angle to a target destination.

- Distance to obstacles detected to the left.

- Distance to obstacles detected by both sensors.

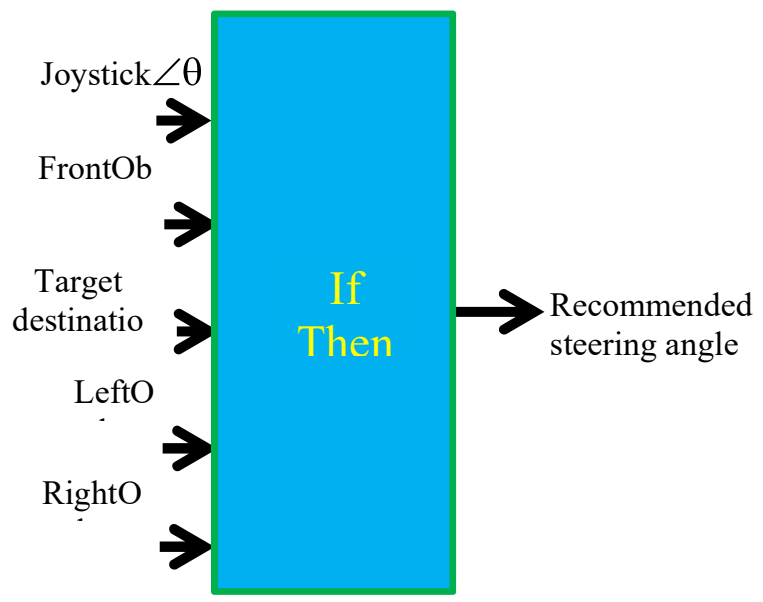

Fig. 3. Revised rule-based expert system.

A volume in front of the chair was described by the ultrasonics. The input from the ultrasonics was used to produce a recommended steering angle that could be used to adjust the angle fed into the wheelchair controller. The new suggested trajectory was safe and efficient. If $\angle \theta$ was right of the chair 
then the chair turned clockwise but if $\angle \theta$ was left of the chair then it turned anticlockwise. The control system improved wheelchair driving if human senses were impaired.

In addition to the joystick steering angle and the volume ahead of the chair, the rule based expert system was also provided with the angle to a target destination. Including that angle to a target destination increased the number of rules but improved operation. Some rules are described as examples:

FIRST EXAMPLE - destination and obstacle to the left:

One: If Joystick $=0^{\mathrm{O}}$ and LeftObs $=\mathrm{HALFWAY}$ and RightObs $\leq$ OUTLYING and FrontObs $\leq$ OUTLYING and AngleToTarget $=75^{\circ}$, then adjust steering angle by $0^{\circ}$

Two: If Joystick $=0^{\mathrm{O}}$ and LeftObs=HALFWAY and RightObs $\leq$ OUTLYING and FrontObs $\leq$ OUTLYING and AngleToTarget $=60^{\circ}$, then adjust steering angle by-10

Three: If Joystick $=0^{\circ}$ and LeftObs=HALFWAY and RightObs $\leq$ OUTLYING and FrontObs $\leq$ OUTLYING and AngleToTarget $=50^{\circ}$, then adjust steering angle by-250

SECOND EXAMPLE - destination and obstacle to the right:

Four: If Joystick $=0^{\circ}$ and LeftObs $\leq$ OUTLYING and RightObs $=$ HALFWAY and FrontObs $\leq$ OUTLYING and AngleToTarget $=75^{\circ}$, then adjust steering angle by $15^{\circ}$

Five: If Joystick $=0^{\circ}$ and LeftObs $=\leq$ OUTLYING and RightObs $=$ HALFWAY and FrontObs $\leq$ OUTLYING and AngleToTarget $=60^{\circ}$, then adjust steering angle by $30^{\circ}$

Six: If Joystick $=0^{\circ}$ and LeftObs $=\leq \mathrm{OUTLYING}$ and RightObs $=$ HALFWAY and FrontObs $\leq$ OUTLYING and AngleToTarget $=30^{\circ}$, then adjust steering angle by $25^{\circ}$

THIRD EXAMPLE - destination to the right and an object in front:

Seven: If Joystick $=0^{\circ}$ and LeftObs $=$ IMMEDIATE and RightObs = IMMEDIATE and FrontObs $\leq$ OUTLYING and AngleToTarget $=20^{\circ}$, then adjust steering angle by $15^{\circ}$

Eight: If Joystick $=0^{\circ}$ and LeftObs $=$ IMMEDIATE and RightObs = IMMEDIATE and FrontObs $\leq$ OUTLYING and AngleToTarget $=25^{\circ}$, then adjust steering angle by $20^{\circ}$

Nine: If Joystick $=0^{\circ}$ and LeftObs $=$ IMMEDIATE and RightObs = IMMEDIATE and FrontObs $\leq$ OUTLYING and AngleToTarget $=30^{\circ}$, then adjust steering angle by $25^{\circ}$
Intelligent Systems Conference 2018 6-7 September 2018 | London, UK

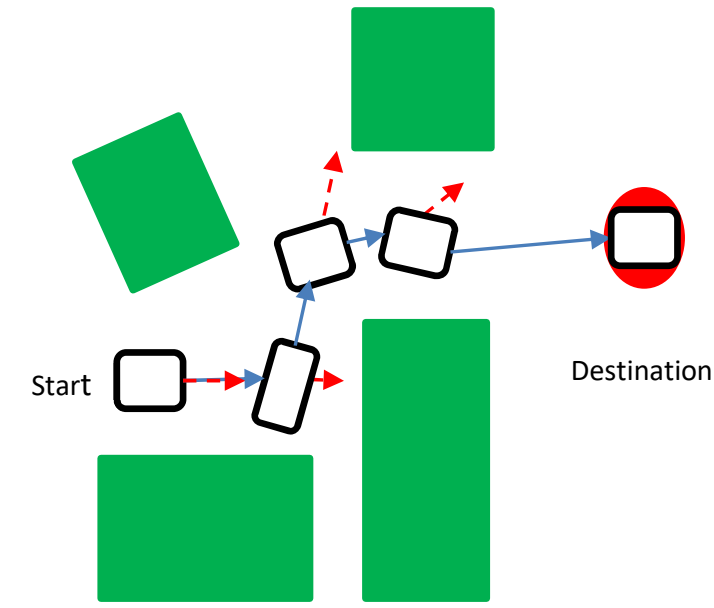

Fig. 4. Use of the rules to guide a powered chair through obstacles. Approach directions (blue solid line) and calculated directions (red dashed line) are shown.

The systems worked well with the revised rules and assisted wheelchair users if a human driver was impaired. The wheelchair path is shown again in Fig. 5 with an additional green solid arrow pointing towards the desired global target destination.

\section{TESTS AND THE RESUlTS}

Fig. 5 shows an example of a simulation. Once the algorithms had been tested successfully during simulations, then the software and hardware were mounted on the chair. Standard test routes at the University of Portsmouth were then used.

If sensors detected an object close to the chair, then the chair tended to turn away in order to avoid a collision. That manoeuvre might be overruled by a wheelchair user by using their joystick if for example they wanted to move close to the object. Typically to reach a wall mounted light switch.

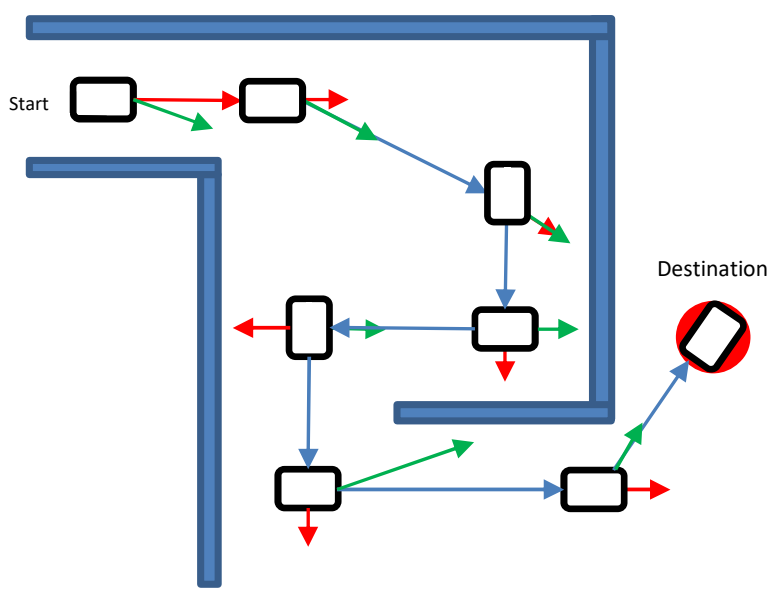

Fig. 5. An example of a simulation showing the wheelchair avoiding local minima (inner wall corners). 


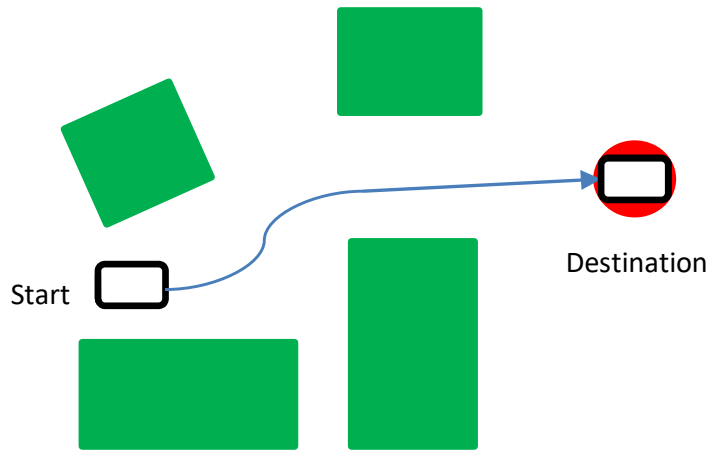

Fig. 6. Results from a real time experiment with the same rules applied.

If an obstacle was detected OUTLYING or nearer then the wheelchair would attempt to avoid the obstacle. If an object was detected ahead of the chair while moving towards the desired target destination, then the chair tended to turn to move alongside the object. When nothing was obstructing the path of the wheelchair and the joystick was held forward, then the chair tended to head for the desired target. Time taken to reach a desired destination tended to be reduced if vision was impaired or if any disability made it difficult to steer. The system would turn the chair to change the bearing so that the chair tended to move in the direction of the desired destination.

Av result from a simulation is shown in Fig. 6 and a result form a real-time test run is shown in Fig. 8.

Results from testing were compared with [3]. This rulebased system tended to complete the standard test routes more quickly. Fig. 7 is a comparison between the time taken by the chair as it was driven through a set of the standard routes at the University of Portsmouth.

In the majority of tests the average time to safely complete a route was less for the systems described in this paper. Fig. 7 shows two anomalies. The chairs completed simple routes (for example routes that were just the empty corridors) without sensors assisting the users. As routes became more complicated, then sensors were useful in assisting users. The addition of a desired target destination as an extra input to the rule based expert system made driving less efficient for easier routes and parts of routes. That was because if a user could see and comprehend what was going on, then they would not need any sensors to help them in a simple uncluttered environment. Examples of standard routes at the University of Portsmouth are represented in Fig. 8 and 9.

In Fig. 8, the rules have a tendency to pull a chair in the direction of the desired target.

Chairs could reach their destinations efficiently. If a powered wheelchair user could steer their wheelchair skilfully then they could overcome the rules if they were making driving less efficient.

The techniques and methods produced faster results for the majority of the routes and reduced computation when compared against other methodologies. The rule-based expert systems were safe and effective.

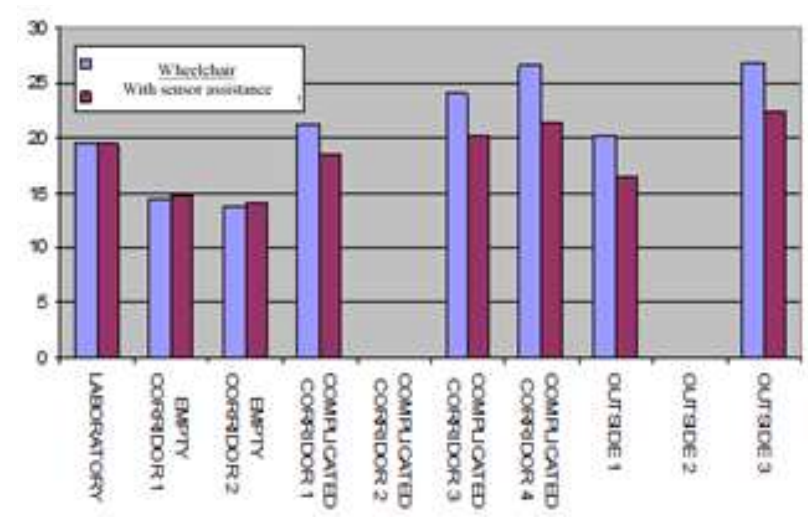

Fig. 7. Average time to complete routes. Right hand (darker) bars are time with sensors assisting a wheelchair driver and left hand (lighter) bars are time taken without sensors assisting.

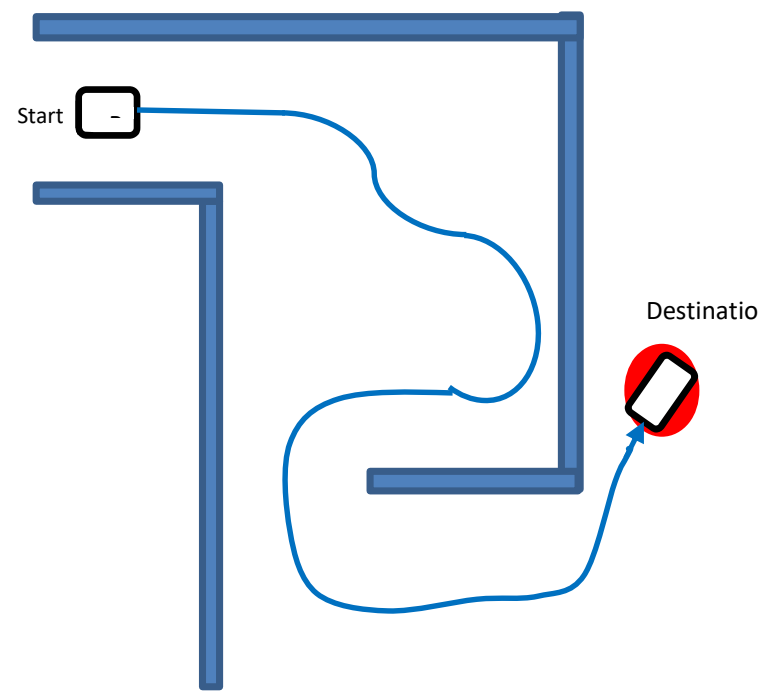

Fig. 8. The path of a wheelchair using the rules when the driver is not able to drive to the destination without assistance.

The chairs had to avoid both stationary and moving obstacles (e.g. human beings moving or standing near the chair).

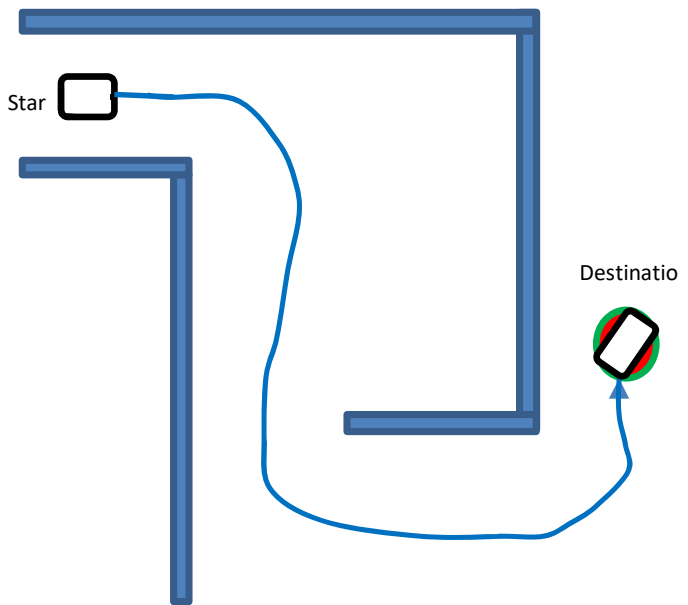

Fig. 9. A wheelchair path when a driver can safely drive themselves to a desired target destination. 
Whenever the sensors detected something near the chair, then the chair was turned to avoid a collision.

The avoidance of collisions is a comparatively high priority. The collision avoidance expert rules tended to override systems but if a joystick was kept stationary in roughly the same place then the user joystick input was integrated over time so that the desire of a disabled driver eventually overrode other behaviour.

If sensor input increased beyond a threshold limit within the array elements then collision avoidance rules were activated.

If an object was detected ahead of the chair while it was moving towards a desired target destination then the chair tended to follow edge of the object; the chair rotated to move parallel to an edge, for example a corridor wall.

If nothing was detected then the chair tended to drive on a bearing between the angle to a desired target and the angle being demanded by the joystick.

Results from testing were compared with results from testing other systems and this rule-based expert system behaved appropriately.

\section{DISCUSSION AND CONCLUSION}

The rule-based expert systems proved to be safe and robust. They were efficient and simple in assisting powered wheelchair user to steer their chair. They successfully assisted drivers by quickly detecting obstacles and safely steering around them. In that way the wheelchair users were assisted to complete routes.

Methods and systems were compared against recently published systems and were validated.

One limitation is that the rules have been hard-coded so that they cannot continue to learn. Future (and some current) research is investigating ways to learn. Current work is also considering mixing AI tools [35]-[49] so that the different AI tools are used to their best effect.

A problem with rule based approaches is that not all environment configurations can be considered at design stage. This raises the question of how the implementation compares with search based control methods, or learnt behaviours (e.g. reinforcement learning, neuro or neuro-fuzzy approaches). A goal-based behaviour could work more efficiently and that will be investigated with other AI techniques in the future?

If the target is an obstacle then the rules would initially try to avoid it. If the driver continues to indicate that they want to drive towards the target (for example a wall to change a lightswitch) then the driver will over-rule the system.

\section{REFERENCES}

[1] Electric Wheelchairs: Types \& Reviews of Powerchairs, Disabled World http://www.disabled-world.com/assistivedevices/mobility/wheelchairs

[2] Mobility Device Statistics - United States - The University of California - Disability Statistics Center - (2013-04-22). http://www.disabledworld.com/disability/statistics/mobility-stats.php

[3] D.R. Parhi, and M.K. Singh "Rule-based hybrid neural network for navigation of a wheelchair," Proc. IMechE Part B: J. Engineering Manufacture, Vol. 224, 2009, pp 11103-1117.
[4] A.V. Nguyen, L.B. Nguyen, S. Su, and H.T. Nguyen "Shared Control Strategies for Human - Machine Interface in an Intelligent Wheelchair" 35th Annual Int Conf of IEEE-Engineering-in-Medicine-and-BiologySociety (EMBC), Osaka, JAPAN. Book Series: IEEE Engineering in Medicine \& Biology Society Conf Proceedings, 2013, pp: 3638-3641.

[5] Z.R. Parhi, S.K. Pradhan, A.K. Panda, and R.K. Behra, "The stable and precise motion control for multiple wheelchairs," Appl. Soft Comput vol 9(2), 2009, 477- 487

[6] D. A. Sanders. B. Sanders, A. Gegov and D. Ndzi, "Results from investigating powered wheelchair users learning to drive with varying levels of sensor support". Proc SAI Intelligent Systems 17

[7] K. T. Song, and C. C. Chen, "Application of asymmetric mapping for wheelchair navigation using ultrasonic sensors," J. Intell. Wheelchairic Syst., 1996, 17(3), 243-264.

[8] R. Huq, G. K. I. Mann and R. G. Gosine, "Wheelchair navigation using motor schema and fuzzy context dependent behaviour modulation," Appl. Soft Comput., 2008, 8(1), 422-436.

[9] M. Begum, G. K. I. Mann, and R. G. Gosine, "Integrated fuzzy logic and genetic algorithmic approach for simultaneous localization and mapping of wheelchairs," Appl. Soft Comput., 2008, 8(1), 150-165.

[10] M. Bennewitz and W. Burgard, "A probabilistic method for planning collision-free trajectories of multiple wheelchairs," In Proc 14th Euro Conf on AI, Berlin, Germany, 20-25 August 2000, pp. 9-15 (ECAI).

[11] W. Gueaieb and M. S. Miah, "An intelligent wheelchair navigation technique using RFID technology," IEEE Trans. Instrum. Measmt, 2008, 57(9), 1908-1917.

[12] C. L. Hwang and N. W. Chang, "Fuzzy decentralized sliding-mode control of a car-like wheelchair in distributed sensor-network spaces," IEEE Trans. Fuzzy Syst., 2008, 16(1), 97-109.

[13] A.V. Nguyen, and L.B. Nguyen, S. Su, H.T. Nguyen, "The Advancement of an Obstacle Avoidance Bayesian Neural Network for an Intelligent Wheelchair", 35th Int Conf of IEEE-Engineering-inMedicine-and-Biology-Society (EMBC), Osaka. Book Series: IEEE Eng in Medicine and Biology Society Conf Proc, 2013, pp: 3642-3645.

[14] D. A. Sanders. "Using a self-reliance factor for a disabled driver to decide on the share of combined-control between a powered wheelchair and an ultrasonic sensor system". IEEE Trans Neural Systems \& Rehab' Eng 25 (8), pp 1221 - $1229 . \quad$ DOI: 10.1109/TNSRE.2016.2620988

[15] D. Sanders, I. Stott, J. Graham-Jones, A. Gegov, and G.E. Tewkesbury, "Expert system to interpret hand tremor and provide joystick position signals for powered wheelchairs with ultrasonic sensor systems". Ind Rob 38 (6). 2011, pp. 585-598.

[16] D.C. Robinson, D.A. Sanders, and E. Mazharsolook. "Ambient intelligence for optimal manufacturing and energy efficiency". Assem. Autom. Vol 35 (3), 201, pp: 234-248

[17] D.A. Sanders, G. Tewkesbury, A. Gegov, et al. "Fast transformations to provide simple geometric models of moving objects". Proc' Intelligent Robotics \& Application Confs, Pt I Vol 9244, 2015, pp: 604-615.

[18] D. Sanders, M. Langner, and G. Tewkesbury, "Improving wheelchairdriving using a sensor system to control wheelchair-veer and variableswitches as an alternative to digital-switches or joysticks", Ind Rob: An int' jnl, 37 (2). 2010, pp. 151-167.

[19] J. Larsson M., Broxvall and A. Saffiotti, "Laser-based corridor detection for reactive Navigation. Ind Rob: An int' jnl, 35 (1), 2008, pp 69-79.

[20] D. Sanders, J. Graham-Jones, and A. Gegov, "Improving ability of teleoperators to complete progressively more difficult mobile robot paths using simple expert systems and ultrasonic sensors," Ind Rob: An int' jnl, 37 (5). 2010, pp. 431-440.

[21] S. Lee "Use of infrared light reflecting landmarks for localization. Ind Rob: An int' jnl 36 (2), 2009, pp: 138-145.

[22] V. Milanes, J. Naranjo, C. Gonzalez, "Autonomous vehicle based in cooperative GPS and inertial systems", Robotica 26, pp 627-633. 2008

[23] D. Sanders and I. Stott, "A new prototype intelligent mobility system to assist powered wheelchair users", Ind Rob 26(6), 09, pp: 466-475.

[24] Y. C. Chang and Y. Yamamoto "On-line path planning strategy integrated with collision and dead-lock avoidance schemes for wheeled 
wheelchair in indoor environments," Ind Rob: An int' jnl, 35 (5), 2008, pp 421-434.

[25] D. Sanders, "Progress in machine intelligence", Ind Rob 35 (6), 2008, pp: $485-487$.

[26] D. A. Sanders, G. Lambert, and L. Pevy, "Pre-locating corners in images in order to improve the extraction of Fourier descriptors and subsequent recognition of shipbuilding parts", Proc' Inst Mech Eng PART B-Jrnl of Engineering manufacture 223, vol 9, 2009, pp: 1217-1223

[27] D. Sanders, "Comparing speed to complete progressively more difficult mobile robot paths between human tele-operators and humans with sensor-systems to assist," Assem. Autom. vol 29 (3), 2009, pp 230-248.

[28] D. Sanders, "Analysis of the effects of time delays on the teleoperation of a mobile robot in various modes of operation," Ind Rob vol 36 Issue: 6, 2009, pp: 570-584. 2.

[29] D. A. Sanders, M. Langner, A. E. Gegov, D. Ndzi, H. Sanders, and G. E. Tewkesbury, G, "Tele-operator performance and their perception of system time lags when completing mobile robot tasks", Proc 9th Int Conf on Human Systems Interaction, pp: 236-242. 2016.

[30] D. A. Sanders, D. L. Ndzi, S. Chester, and M. Malik, "Adjustment of tele-operator learning when provided with different levels of sensor support while driving mobile robots", IEEE Proc' of SAI Intelligent Systems, pp: 962-967. 2016.

[31] D. A. Sanders and N.Bausch, (Ed H. Liu). "Improving Steering of a Powered Wheelchair Using an Expert System to Interpret Hand Tremor". Proc of Intelligent Wheelchairics and Applications (Icira 2015), Pt Ii Volume: 9245, pp: 460-471. 2015

[32] D. Sanders, I. J. Stott and D. C. Robinsosn et al. "Analysis of successes and failures with a tele-operated mobile robot in various modes of operation". Robotica 30, pp: 973-988. 2012.

[33] D. A. Sanders, G. E. Tewkesbury, and I. J. Stott, et al. "Simple expert systems to improve an ultrasonic sensor-system for a tele-operated mobile-robot". Sensor Review 31 (3), pp 246-260. 2011.

[34] G. Eason, B. Noble, and I. N. Sneddon, "On certain integrals of Lipschitz-Hankel type involving products of Bessel functions," Phil Trans. Roy. Soc. London, vol. A247, pp. 529-551, April 1955.

[35] D. A. Sanders, H. Sanders, D. L. Ndzi, and A. E. Gegov, "Rule-based system to assist a tele-operator with driving a mobile robot", IEEE Proc' of SAI Intelligent Systems Conf', pp: 987 - 994. 2016.

[36] D. Sanders and A. Geov. "AI tools for use in Assembly Automation and some examples of recent applications. Assem. Autom. Vol 33 (2), pp: 184-194. 2013.

[37] D. A. Sanders, G. E. Tewkesbury, and D. Ndzi et al. "Improving automatic robotic welding in shipbuilding through the introduction of a corner-finding algorithm to help recognise shipbuilding parts". Journal of Marine Science and Technology Vol 17 (2), pp: 231-238. 2012.

[38] D. Sanders, G. Lambert, and J. Graham-Jones et al. "A robotic welding system using image processing techniques and a CAD model to provide information to a multi-intelligent decision module". Assem. Autom. Vol: 30 (4), pp: 323-332. 2010.

[39] D. A. Sanders, and G. E. Tewkesbury. "A pointer device for TFT display screens that determines position by detecting colours on the display using a colour sensor and an Artificial Neural Network" Displays Vol 30 (2), pp: 84-96. 2009.

[40] J. Bergasa-Suso, D. A. Sanders, and G. E. Tewkesbury. "Intelligent browser-based systems to assist Internet users". IEEE Transactions on Education Vol 48 (4), pp: 580-585. 2005

[41] D. A. Sanders, Y. Tan, I Rogers et al. "An expert system for automatic design-for-assembly". Assem. Autom. 29 (4), pp: 378-388. 2009.

[42] D. A. Sanders, G. Lambert, and L. Pevy. "Pre-locating corners in images in order to improve the extraction of Fourier descriptors and subsequent recognition of shipbuilding parts". Proc of IMechE Part BJournal of Engineering Manufacture 223 (9), pp: 1217-1223. 2009.

[43] D. Sanders. "Controlling the direction of walkie type forklifts and pallet jacks on sloping ground". Assem. Autom. 28 (4), pp: 317-324. 2008

[44] D. A. Sanders, J. Bergasa-Suso, R. Khusainov, A. Gegov, S. Chester, and N. Bausch. "Introducing dead bands within two-dimensional clusters of user data to improve data classification". Proc' of the IEEE 9th Int Conf on Human Systems Interaction, pp: 14-20. 2016.

[45] D. A Sanders"Progress in machine intelligence". Ind Rob: An int' jnl 35 (6), pp: 485-487. 2008.

[46] A. Geov, N. Gobalakrishnan and D. A. Sanders. "Rule base compression in fuzzy systems by filtration of non-monotonic rules". Journal of Intelligent \& Fuzzy Systems Vol 27 (4), pp: 2029-2043. 2014.

[47] D. A Sanders, A. Gegov and D. Ndzi. ", Knowledge-based expert system using a set of rules to assist a tele-operated mobile robot. Y Bi, S Kapoor \& R Bhatia (eds), Studies in Computational Intelligence, vol. 751, Springer, pp. 371-392. 2018.

[48] D. A. Sanders, H. Sanders, A. Gegov and D. Ndzi. "Rule-based system to assist a tele-operator with driving a mobile robot". Lecture Notes in Networks and Systems, vol. 16, Springer, pp. 599-615. 2018,

[49] D. A. Sanders. 'Non-model-based control of a wheeled vehicle pulling two trailers to provide early powered mobility and driving experiences' IEEE Transactions on Neural Systems and Rehabilitation Engineering, vol 26, no. 1, pp. 96 - 104. 2018. 